\title{
COVID-19: The CaMKII_Like System of S Protein Drives Membrane Fusion and Induces Syncytial Multinucleated Giant Cells
}

\author{
Wenzhong Liu ${ }^{1,2, *}$, Hualan $\mathrm{Li}^{2}$ \\ 1 School of Computer Science and Engineering, Sichuan University of Science \& Engineering, \\ Zigong, 643002, China; \\ 2 School of Life Science and Food Engineering, Yibin University, Yibin, 644000, China; \\ * Correspondence.Wenzhong Liu, liuwz@suse.edu.cn.
}

\begin{abstract}
COVID-19 is a unique disease characterized by extensive pulmonary thrombosis and infected syncytial multinucleated giant cells, relating to extensive tissue damage. The SARS-CoV-2 S protein on the membrane of infected cells can initiate receptor-dependent syncytia formation. To study the membrane fusion on S protein, we adopted structural domain search methods to analyze the structural and non-structural proteins of the SARS-COV-2 virus in this study. The results showed that the surface glycoprotein (S) had conserved domains of CaMKII: CaMKII_AD, CaATP_NAI, DUF4440, EF-hand, Protein kinase, and SnoaL-like. Comparing to SARS-COV and MERS, only the CaATP_NAI of SARS-COV-2 is in the contact position of the viral membrane and cell membrane. We believed that when the EF-hand domain ("YEQYIKWPWYIWLGF") of $\mathrm{S}$ protein bound to calcium ions, S2 protein had CaMKII protein activities. After the S protein fusion peptide was inserted into the infected cell membrane and fixed the S2 protein on the cell membrane, the CaMKII_AD prompted the S2 protein to form HR1-HR2 six-helix bundles. The HR1-HR2 hexamer had three CaATP_NAI domains (“APAICHDGKAHFPRE”) near the viral membrane (contact position), the CaATPase activated by magnesium ions, and released energy through ATP phosphorylation. The CaATPase drove the HR1-HR2 hexamer fold irreversibly toward the viral membrane. Then the CaATP_NAI and CaMKII_AD domains extended to the outside and combined the viral membrane and the cell membrane so that the contact position formed a thin barrel structure. The hydrated calcium ions are gathered in the barrel structure to create a calcium bridge. The release action of water in contact position caused the instability of the double membrane, triggering lipid mixing and fusion of the membrane. CaATPases disassembled the barrel structure, and HR1-HR2 hexamer is fell into the cytoplasm. The viral membrane fused with the cell membrane on a large scale. The cytoplasmic contents of the virus mixed with the cell. The S protein of the infected cell may bind to the ACE2 receptor of another cell (or also an infected cell) and then achieved membrane fusion through a similar principle, forming cell syncytia, includes syncytial multinucleated giant cells. The membrane fusion could disrupt the calcium homeostasis in human body, and increased the risk of coagulation and vascular calcification.
\end{abstract}

Keywords: Cell syncytia; Syncytial multinucleated giant cells; CaATPase; HR1-HR2 Hexamer; Calcium homeostasis; 


\section{Background}

COVID-19 is a unique disease characterized by extensive pulmonary thrombosis, the presence of viral RNA in lung cells and endothelial cells, and the formation of infected cell syncytia(1). Histopathological results show that the patient's lungs are present interval lymphocyte infiltration, macrophage alveolar exudate, the cells are enlarged, and the cytoplasm and lysosomes are significantly enlarged(2), focal type II pulmonary cell hyperplasia, multinucleated syncytial cell-induced cytopathic changes(3). Atypical mesothelial cell aggregates with enlarged nuclei and multinucleated syncytial cells also are found in the pleural effusion cell by blocking agent preparations(4). Alveolar syncytial cells have large nucleoli, granular cytoplasm, and prominent nucleoli(5). The SARS-CoV-2 $\mathrm{S}$ protein on the membrane of infected cells can initiate receptor-dependent syncytia formation, relating to extensive tissue damage(6). However, the mechanism by which the SARS-CoV-2 S protein regulates the formation of these syncytia is not fully clear. A study has found that SARS-CoV-2 S protein mediated lymphocyte elimination through syncytia(7). Infected cells form syncytia through membrane fusion with minimal $\mathrm{S}$ protein, making be challenging to obtain neutralizing antibodies and prevent them through antibodies effectively(8). Therefore, understanding the mechanism of SARS-CoV-2 S protein driving membrane fusion and inducing syncytia is of great value for immune prevention and treatment.

The lungs of COVID-19 patients show inflammatory infiltration and plaque fibrin deposition, and prominent fibroblast foci are related to occasional alveolar macrophages. Biopsy of the patients reveals lung cell damage and shedding, atypical pneumonia cells with large nuclei( 9 ), focal lymphocyte infiltration, and the formation of syncytial giant cells(10). In syncytial multinucleated giant cells, no viral inclusions or squamous metaplasia are observed(11). Syncytial giant cells show depletion of nuclear chromatin and have a peripheral nuclear membrane (12). In the SARS-CoV-2 histological experiments on rhesus monkeys(13) and cynomolgus monkeys(14), multinucleated syncytial cells of epithelial origin are evident the airways and parenchyma. In the lymphocyte infiltration of lung syncytium, giant multinucleated cells are also frequently detected. However, these cells express lung differentiation marker TTF-1 instead of macrophage markers such as CD68(15). Syncytial respiratory virus, metapneumovirus, paravirus, adenovirus, boca virus, coronavirus, and mycoplasma pneumoniae infection are tested negative for respiratory secretions on admission(16). The syncytia of COVID-19 patients may be attributed to SARS-CoV-2 condition.

The SARS-CoV-2 virus triggers cell fusion between the infected and neighboring target cells to form an enlarged cell hybrid or syncytia. Early theories believe that the formation of multiple nuclei in syncytia may represent virus-induced cytopathics, resulting from SARS-CoV-2's clearance of lung cell nuclei(12). Since the SARS-CoV-2 virus adopts cytoplasmic mechanisms to replicate without entering the nucleus of susceptible cells (such as type II lung cells), this classical nuclear viral cytopathy may not be a suitable SARS-CoV-2 virus(17). Experiments have shown that deleting the ER retention motif on the SARS-CoV-2 S protein can reduce cell hybridization during cell-cell fusion(6). In addition to granular cell fusion, $\mathrm{S}$ also mediates the fusion between infected and uninfected cells, leading to syncytia formation. The $\mathrm{S}$ protein that is not assembled into a virion is transferred to the plasma membrane. It mediates fusion between cells, inducing in a vast multinucleated syncytium(18). Areas where infected cells tend to form clusters and adhere to 
each other, forming giant cell-like syncytial aggregates, multinucleated syncytial cells(19).

In vitro experiments show living cells infected with SARS-CoV-2 also form typical syncytia(20). S protein effectively mediates the formation of syncytia between effector and target cells without exogenous proteolytic enzymes(21). It shows that SARS-CoV-2 may mainly use the plasma membrane fusion pathway to enter the host cell and replicate in the host cell. Virus and cell fusion is through protein-lipid or protein-protein interaction. The protein activity involved in the fusion makes the viral membrane and the cell membrane more tightly bound(22). Fusion peptide of viral protein actively destroys the lipid organization in the membrane, facilitating fusion events(23). Many factors cause an irreversible conformational change of viral protein, and then the fusion protein folds back(24). Some experiments can induce liposomes to fuse without protein. Once the two membranes are very close, lipid mixing will occur between the proximal membrane lobules and then between the distal lobules, causing the cytoplasm between the two cells to connect(25).

The salient feature of the SARS-CoV-2 S peak is the presence of multi-base cleavage sites. A recent CRISPR-Cas9 screen shows that the entry of SARS-CoV-2 into the cell line appears to be mediated by endosomal cathepsin(26). The entry of SARS-CoV-2 into human airway organoids is mediated by serine proteases and promoted by multi-base cleavage sites (MBCS). S protein comprises two domains, namely receptor binding (S1) domain and fusion (S2) domain. These domains are separated by S1/S2 cleavage sites. Membrane fusion is determined by the double arginine motif in the S1/S2 cleavage site, which is usually present in the surface glycoproteins of most highly infectious viruses. The multi-base cleavage site contains a proline-arginine-arginine-alanine (PRRA) motif(27). MBCS speeds up viral entry and promotes cell fusion. SARS-CoV-2 S Replacement of MBCS with SARS-CoV's one site reduces the fusion of monkey kidney cell line (VeroE6) and the infectivity of human lung adenocarcinoma cell line (Calu-3)(28). The inhibitory effect of serine proteases reduces replication of SARS-CoV-2 virus(29). Serine protease inhibitor (camostat mesylate) also prevents SARS-CoV-2 pseudo-particles from entering Calu-3 cells(30). TM protease serine 2 (TMPRSS2) is a type 2 TM serine protease on the host cell membrane(31). TMPRSS2 is a calcium-dependent serine protease that can cleave $\mathrm{S}$ at $\mathrm{S} 1 / \mathrm{S} 2$ site and accelerate the fusion between SARS-CoV-2 and infected cell(32).

Surface Glycoprotein (S protein) of SARS-CoV-2 has an RGD motif in its receptor-binding domain(RBD). The RGD motif governs the role of cell attachment and cell adhesion. The calculation results show that there are calcium and metal-binding sites around and near the RGD-integrin docking site. The RGD-integrin interaction may occur in a calcium-dependent manner(33). The general molecular mechanism of membrane fusion involves $\mathrm{Ca}^{2+}$. There is a set of specialized proteins in the opposite membrane bilayer for membrane fusion(34). It involved calmodulin in extracellular/endocytosis and endosomal antigen activity(35). Calmodulin (CaM) is a multifunctional protein in various eukaryotic cells and can bind to calcium ions. Calmodulin plays a crucial role in the $\mathrm{Ca}^{2+}$-dependent signal transduction pathway. It is a dynamic $\mathrm{Ca}^{2+}$ sensor that responds to a wide range of $\mathrm{Ca}^{2+}$ concentrations and transmits signals downstream(36). Platelets in the serum(37) release-activated calmodulin(38). CaMKII is a vital calmodulin effector for endosomal fusion(39). CaMKII is activated by $\mathrm{Ca}^{2+}$-bound calmodulin $\left(\mathrm{Ca}^{2+} / \mathrm{CaM}\right)$, and this activation is related to the frequency(40) and positional signals(41) of $\mathrm{Ca}^{2+}$. CaMKII is also a multifunctional serine/threonine kinase expressed abundantly in the heart. A variety of upstream 
signals activates CaMKII, including $\mathrm{Ca}^{2+}$ and $\mathrm{ROS}(43)$. Long-term $\mathrm{Ca}^{2+}$ pulses and ROS convert CaMKII into autonomous enzymes that do not rely on $\mathrm{Ca}^{2+}$ and calmodulin.

Calcium triggers protease-mediated membrane fusion activation of SARS-COV-2(42). The S1 subunit contains a receptor-binding domain that recognizes and binds to the host receptor Angiotensin-Converting Enzyme 2. In contrast, the S2 subunit mediates viral cell membrane fusion by forming a six-helix bundle through the two-seven repeat domains(43). The insertion of the fusion peptide (FP) of S2 into the cell membrane starts a six-helix bundle conformation and prompts membrane fusion(44). The fusion peptide binds two $\mathrm{Ca}^{2+}$ ions, and the two $\mathrm{Ca}^{2+}$ binding sites show greater synergy(45). The combination of $\mathrm{Ca}^{2+}$ ions with the FP can enhance penetration. FP penetration can change the molecular organization of a specific area of the double layer. In the absence of $\mathrm{Ca}^{2+}$ ions, membrane binding of FP will be significantly reduced(46). There are phosphorylated residues Y789 and T791(47) near the N-terminus of FP, which may increase the negative charge and facilitate the accumulation of $\mathrm{Ca}^{2+}$ ions. Beside, JPT2 is a component of the NAADP receptor complex and is essential for TPC-dependent $\mathrm{Ca}^{2+}$ signaling and coronavirus entry control(48). TPC is a channel in the endolysosome system. When the intracellular messenger NAADP (niacin adenine dinucleotide phosphate ) activates TPC, it released $\mathrm{Ca}^{2+}$ through the TPC channel. In the process of SARS-CoV-2 fusions, NAADP mediates $\mathrm{Ca}^{2+}$ signaling. Acidic endosomes and TPC promote the release of $\mathrm{Ca}^{2+}$ from the endolysosome system(49).

If the $\mathrm{S}$ protein mediates the SARS-CoV-2 virus and cell membrane fusion in a calcium-dependent manner, the demand for $\mathrm{Ca}^{2+}$ is enormous. So the $\mathrm{Ca}^{2+}$ homeostasis in the human body is easily destroyed. For COVID-19 patients, calcitonin(PCT), C-reactive protein(CRP), and neutrophil to lymphocyte ratio (NLR) indicators are elevated in moderate and severe patients (50). Calcitonin is a hormone that regulates blood calcium secreted by the thyroid and bronchi. It responds to hypercalcemia. It quickly lowers blood calcium by inhibiting bone resorption $(51,52)$. There is also a report showing that the total serum calcium and the actual ionized calcium levels of the whole blood of COVID-19 patients are lower(53). COVID-19 patients have low serum zinc, calcium, and vitamin D levels(54). Hypocalcemia is an essential and reliable indicator of disease severity and progression, but vitamin D levels cannot reflect the correlation with the severity of COVID-19 infection (55). It indicates that there may be a disorder in the calcium regulation system of COVID-19 patients(56).

Most viral infections can cause changes in intracellular $\mathrm{Ca}^{2+}$ concentration. $\mathrm{Ca}^{2+}$ plays a vital role in the process of virion structure formation, virus entry, virus gene expression, virus protein translation, virus particle maturation, and release(57). Most known mechanisms indicate viruses interfere with $\mathrm{Ca}^{2+}$ stability and facilitate virus replication by interfering with $\mathrm{Ca}^{2+}$ and $\mathrm{Ca}^{2+}$ binding proteins(58). Viral proteins interfere with $\mathrm{Ca}^{2+}$ homeostasis by changing membrane permeability or manipulating key components of $\mathrm{Ca}^{2+}$ signaling pathway(57). Viruses provide enough space for processing the gradient of the membrane interval, and can easily convert various signals. To live in the host cell, the virus hijacks important $\mathrm{Ca}^{2+}$ signaling pathways(59). Viruses also speed up or induce cell apoptosis by interfering with the $\mathrm{Ca}^{2+}$ pathway to promote the release of virus particles and spread the virus to the greatest extent $(60)$.

In this study, we adopted bioinformatics techniques such as conserved domain search to study the calmodulin or CaMKII regulation-related functions of the SARS-COV-2 virus protein. The results showed that the $\mathrm{S}$ protein had CaMKII conserved domain. EF-hand domain ("YEQYIKWPWYIWLGF") of S protein bound to calcium ions, S2 protein had both calmodulin 
and CaMKII protein activities. After the $\mathrm{S}$ protein fusion peptide was inserted into the infected cell membrane and fixed the S2 protein on the cell membrane, the CaMKII_AD prompted the S2 protein to form HR1-HR2 six-helix bundles. There are three CaATP_NAI domains ("APAICHDGKAHFPRE", CaATPase) on the membrane contact surface of the six-helix. They are powered by ATP to promote the irreversible folding of the six helix, drivinging the precise fusion of the viral membrane and the cell membrane. Therefore, the CaMKII_like system of S protein membrane fusion to induce syncytial multinucleated giant cells..

\section{Method}

\subsection{Data set}

1. The sequences of SARS-COV-2 proteins. The SARS-COV-2 protein sequences came from the NCBI database. Including: S, E, N, M, ORF3a, ORF8, ORF7a, ORF7b, ORF6, ORF10, orflab. In addition, we also downloaded the S protein sequence of SARS-COV and MERS virus from NCBI for comparison with SARS-COV-2 protein.

2. Bacterial calmodulin-related sequence. We downloaded 6386 protein sequences of bacterial calmodulin-related from the UniProt data set and searched keyword was "Bacterial + Calmodulin". The calmodulin-related sequences were compared with the viral proteins to search for the conserved domains.

3. Crystal structures of HR1-HR2. we downloaded the HR1-HR2 (PDB ID:6m3w) protein from the PDB database.

\subsection{Localized MEME tool to scan for conserved domains.}

The analysis steps are listed as follows:

1. Download MEME from the official website and subsequently install in the virtual machine ubuntu operating system. The virtual machine was VM 15.2.

2. Download the SARS-COV-2 protein sequence of SARS-CoV-2 from NCBI official website.

3. Download the fasta format sequence of calmodulin-related from Uniprot official website, respectively. The search keyword was "bacteria + calmodulin" .

4. For each sequence in all calmodulin-related protein, paired with each SARS-COV-2 protein sequence to generate fasta format files for MEME analysis.

5. For the files generated in Step 4, a batch of 50000 was used to create several batches. It was considered as the limited space of the virtual ubuntu system.

6. In ubuntu, searched the conserved domains (E-value $<=0.05$ ) of SARS-COV-2 protein and calmodulin-related with MEME tools in batches.

7. Collected the result files of conserved domains. Find the domain name corresponding to the motif from the uniprot database.

8. We analyzed the domains' activity of the each SARS-COV-2 protein according to the characteristics of the calmodulin-related protein domains.

\subsection{Multiple sequence alignment}

We used the local version of ClustalX2 to perform multiple sequence alignments on the $\mathrm{S}$ proteins of SARS-COV-2, SARS-COV and MERS. The parameters are the default values. 


\section{Results}

\subsection{S protein had conserved domains of CaATP_NAI and CaMKII_AD}

The structure of the functional CaMKII enzyme is a dodecamer as two stacked six-mer rings (61). Each monomer comprises an N-terminal catalytic domain and a C-terminal association domain. Between the two, it also includes the auto-regulatory domain (auto-inhibitory peptide, AIP) of the $\mathrm{Ca}^{2+} / \mathrm{CaM}$ binding site, which regulates the activation state through $\mathrm{Ca}^{2+} / \mathrm{CaM}^{2}$ binding and autophosphorylation(62). The $\mathrm{Ca}^{2+} / \mathrm{CaM}$-dependent activation of CaMKII depends on the total $\left[\mathrm{Ca}^{2+}\right]$ in a dose-dependent manner, and the frequency, amplitude, and duration of the $\mathrm{Ca}^{2+}$, as well as the previous activation state(63). When inactive, the regulatory domain sterically encloses the catalytic domain. This mode is also called the self-inhibition state. When the $\mathrm{Ca}^{2+} / \mathrm{CaM}$ binds autoregulatory region, the kinase is activated, resulting in a conformational change. It exposes the kinase substrate site of the catalytic domain and adenosine triphosphate (ATP) binding site(64). When a monomer enters the active state, it used the regulatory domain of the adjacent CaMKII monomer for autophosphorylation at Thr287 to promote the activation of CaMKII further. It prevents the catalytic domain and self-inhibition domain. Even in the dissociation of the $\mathrm{Ca}^{2+} / \mathrm{CaM}$ complex, the kinase activity remains. At higher stimulation frequencies, autophosphorylation of key threonine residues next to AIP (such as Thr 287 in cardiac $\delta$ subtype) makes following kinase activity independent of $\mathrm{Ca}^{2+}$ and $\mathrm{CaM}(65)$. Autophosphorylation of $\mathrm{Thr}$ 305 and Thr 306 in AIP prevents CaM binding and subsequent kinase reactivation(62). And the affinity of the kinase to $\mathrm{CaM}$ is significantly increased, and the $\mathrm{Ca}^{2+} / \mathrm{CaM}$ complex is kept in the proper position to maintain the activity of CaMKII under low $\left[\mathrm{Ca}^{2+}\right]$ conditions(66).

CaMKII_Like domains of $\mathbf{S}$ protein. We downloaded the bacterial calmodulin-related protein sequence from the UniProt website (search keyword "bacteria + calmodulin" ). We then used the MEME local tool to compare each protein sequence of the virus with the calmodulin-related protein sequence, and searched for conserved domains (table 1). Table 1 shows that all proteins have the CaMKII_AD and Protein kinase domain. Many proteins have SnoaL-like, and EF-hand domains. We integrated the S protein motif sequences by domain and continuity (Table 2). Table 2 shows that S protein has CaMKII_AD, CaATP_NAI, DUF4440, EF-hand, Protein kinase, SnoaL-like domains. The catalytic domain of CaMKII protein binds ATP. In Table 1, only S protein has the CaATP_NAI domain---a domain that can bind to ATP. It shows that only S protein may have similar activity to the CaMKII protein. From the KEGG official website, we found that the Mus musculus CaMKII protein with entry=12323. Its motifs are Pkinase, CaMKII_AD, PK_Tyr_Ser-Thr, DUF4440, SnoaL_3, SnoaL_2 domains (E value $<0.05$ ). According to Table 1, it can be seen that $\mathrm{S}$ has other domains except for the PK_Tyr_Ser-Thr domain.

The $\mathrm{S}$ protein of coronavirus contains the $\mathrm{S} 1$ subunit (at N-terminal) that mediates the recognition of host cell receptors. The subunit $\mathrm{S} 2$ (at C-terminal) fuses the viral membrane with its host cell(67). The RBD domain of the $\mathrm{S}$ protein has different conformations and corresponding functions in the open-and-close states. The $\mathrm{S}$ protein has a furin cleavage site at the boundary between S1/S2(68). TMPRSS2 starts the $\mathrm{S}$ protein, which requires two potential sites, Arg685/Ser686 and Arg815/Ser816, to cleave S proteins (69). After cleavage at the S1 and S2 subunits boundary, the $\mathrm{S}$ protein keeps the trimer by non-covalent binding. Based on the coronavirus S protein monomer structure, the S1 and S2 subunits form the spherical head and stem 
regions $(70)$. The S2 trimer is conserved in coronaviruses. It undergoes a series of conformational changes to trigger membrane fusion between the host cell membrane and the viral envelope(71). S2 has low variability(72), and the structure and function of HR1 - HR2 six-helix bundles are also highly conserved(73). So the HR domain is now also an ideal target for SARS-CoV-2 infection inhibitors. Table 2 shows that CaMKII-like related domains are mainly in and near the S2 protein region. But we did not find that these two cleavage sites are located in the CaMKII-like related domains of the $\mathrm{S}$ protein. It indicates that CaMKII-like related domains may have relatively conservative functions.

Table 1. CaMKII-like related domains possessed by the SARS-COV-2 virus protein

\begin{tabular}{lccccccccccc}
\hline \multicolumn{1}{c}{ Domain } & S & N & E & M & orflab & ORF3a & ORF6 & ORF7a & ORF7b & ORF8 & ORF10 \\
\hline CaMKII_AD & V & V & V & V & V & V & V & V & V & V & V \\
CaATP_NAI & V & & & & & & & & & & \\
DUF4440 & V & & & & & & & & & & \\
EF-hand & V & & & & V & V & V & & V & & V \\
Protein kinase & V & V & V & V & V & V & V & V & V & V & V \\
SnoaL-like & V & V & & & V & V & & & & & \\
\hline
\end{tabular}

Table 2. CaMKII-like related motifs possessed by S protein of SARS-COV-2

\begin{tabular}{|c|c|c|c|}
\hline Domain & Motif & Start & End \\
\hline CaATP_NAI & APAICHDGKAHFPRE & 1078 & 1092 \\
\hline \multirow[t]{6}{*}{ CaMKII_AD } & AQEKNFTTAPAICHDGKAHFPREGVFVSNGTHW & 1070 & 1102 \\
\hline & CNDPFLGVYYHKNNKSWMESEFR & 136 & 158 \\
\hline & ELGKYEQYIKWPWYIWLGFIAGLIAIVMVTIMLCCMTSCCSCLKGCCSCGSCCKF & 1202 & 1256 \\
\hline & MQMAYRF & 900 & 906 \\
\hline & RFASVYAWNRKRI & 346 & 358 \\
\hline & RVDFCGKGYHLMSFPQSAPH & 1039 & 1058 \\
\hline DUF4440 & QYIKWPWYIW & 1208 & 1217 \\
\hline EF-hand & YEQYIKWPWYIWLGF & 1206 & 1220 \\
\hline Protein kinase & YEQYIKWPWYIW & 1206 & 1217 \\
\hline \multirow[t]{2}{*}{ SnoaL-like } & WPWYIW & 1212 & 1217 \\
\hline & YHKNNKSWMES & 145 & 155 \\
\hline
\end{tabular}

X. Fan et al. determined the S2 structure before and after the fusion (74). The transition from the pre-fusion state to the post-fusion state triggers the drastic structural rearrangement and conformational changes of SARS-CoV S glycoprotein, and the largest structural rearrangement is located in the HR1-CH helix region. By comparing the sequence's range, we found that the CaMKII_AD and CaATP_NAI domains play an essential role in the fusion. The CaMKII_AD motif "MQMAYRF" (900-906) is in the HR1 motif(894-966) region, and the CaMKII_AD motif “RVDFCGKGYHLMSFPQSAPH” (1039-1058) is in the BH motif (1017-1053) region. The CaMKII_AD motif “AQEKNFTTAPAICHDGKAHFPREGVFVSNGTHW” (1070-1102) is in the SD3 motif (1054-1104) region, and the CaATP_NAI domain “APAICHDGKAHFPRE” (1078-1092) is also in the SD3 motif. HR1 is a crucial component of HR1-HR2 six-helix. The pan-coronavirus fusion inhibitor EK1 peptide variant EK1C4 can inhibit SARS-CoV-2 fusion in a 
dose-dependent manner by binding to HR1(21). The BH motif generates $\beta$-hairpin. X. Fan et al. believes that the linker region and the SD3 domain are involved in the folding and extension actions during fusion. The disulfide bond (C1064 - C1108) between the linker region upstream of the HR2 motif and the SD3 domain stabilizes the linker region during the fusion transition. The disulfide bond is in the overlapping region of the CaMKII_AD and CaATP_NAI domains. Except FP motif (789-809, fusion peptide), CR motif (823-893, connecting region), CH motif (967-1016, central helix region), L motif (688-711, 1105- 1143,), UH motif (712-753, upper spiral region), HR2 motif (1145-1178), the CaMKII_AD domain makes up three motifs of HR1, BH and SD3. It means that HR1, BH, and SD3 mediate the membrane fusion process through activity of CaMKII_AD and CaATP_NAI.

CaMKII_AD domain. The concentration of $\mathrm{Ca}^{2+}$ usually increases during stress, which can be sensed by sensor proteins or calcium-binding proteins (CBP), which initiate a signaling cascade for adaptation(75). CAMKII has a self-associative domain (CaMKII_AD) found at the C-terminus, which helps to assemble a single protein into two stacked hexameric holoenzymes composed of two monomers(61). The calmodulin-responsive regulatory fragment forms coiled-coil support, which prevents the peptide and ATP from binding to the kinase domain that initially has intrinsic activity. After the threonine residues in the regulatory chain is phosphorylated, the CaMKII's calmodulin binding domain is independent, separated from the catalytic site by the organization of the dimer. It ensures a strict $\mathrm{Ca}^{2+}$ dependency for initial activation.

Once the S1 subunit of the S protein binds to the host cell ACE2, the fusion peptide (FP) is inserted into the target cell membrane. Then it exposes the HR1 domain's hairpin pre-coiled coil, triggering the interaction between HR1 and HR2. It exposed three high conserved hydrophobic grooves on the surface of HR2, forming the HR1-HR2 six-helix bundle when helices and loops to interact with the HR1 domain(44). The S2 subunit of the S protein forms the HR1-HR2 six-helix bundle, which is essential for virus fusion(76). The junction region upstream of the HR2 motif is critical for creating the HR1-HR2 six-helix bundle(74). HR2 is at residues 1163-1213, and part of the fragment is in the C-terminal CaMKII_AD domain of the $\mathrm{S}$ protein ("ELGKYEQYIKWPWYIWLGFIAGLIAIVMVTIMLCCMTSCCSCLKGCCSCGSCCKF", 1202-1256) region. The C-terminal CaMKII_AD domain is in and near the virus cytoplasmic domain(77). The cytoplasmic domain region is close to the viral membrane-proximal part of the viral membrane $\mathrm{CD}$ domain region (1234-1273). It shows a conserved cysteine abundance, and "CSCLKGCCSCGSC" is the consensus motif region(78). The consensus pattern is $\mathrm{C}$-[TS]-C-h-X-G-X(4,6)-C, CAF-motif (cysteine aggregation fusion), where $\mathrm{h}$ is a hydrophobic residue and $\mathrm{X}$ is any other residue. This specific domain mediates the particle assembly of coronavirus spikes(78). The cysteine-rich region facilitates the formation of fusion pores(79). The importance of its membrane fusion has also been confirmed(80). The palmitoylation of the cysteine and its internal domain is also the rate-limiting step for changing the membrane fusion reaction(81). It finds similar functional cysteine structures in the proteins of influenza virus HA, vesicular stomatitis virus, influenza virus and murine leukemia virus(78). There are also three adjacent CaMKII_AD domains in the connecting region upstream of the HR2 motif: (“MQMAYRF”, 900-906), (“AQEKNFTTAPAICHDGKAHFPREGVFVSNGTHW”, 1070-1102) and ("RVDFCGKGYHLMSFPQSAPH", 1039-1058).

It shows that the CaMKII_AD domain of the S protein is involved in the formation of the HR1-HR2 six-helix bundle, holding the function of the self-association domain of the CAMKII 
protein. Interestingly, the crystal structure of N, E, ORF8 and ORF3 proteins also are multimeric structures. These proteins all have the CaMKII_AD domain. The formation of multimers through the CaMKII_AD domain may be an essential way for the SARS-COV-2 virus.

CaATP_NAI domain. CaATP_NAI is ACAs (auto-inhibited calcium ATPases), which is like "plasma membrane-type" $\mathrm{Ca}^{2+}$-ATPase $\left(\mathrm{Ca}^{2+}\right.$ pump, ACA2). This type IIB $\mathrm{Ca}^{2+}$ ATPase exists in the plasma membrane (PM) of animals and plants(82). CaATP_NAI lacks the long C-terminal calmodulin binding regulatory domain(83), is insensitive to calmodulin(84), and does not require calmodulin to activate the $\mathrm{Ca}^{2+}$ pump. Although CaATP_NAI is small, the truncated pump is fully active. CaATP_NAI $\left(\mathrm{Ca}^{2+}\right.$-ATPase) is the N-terminal self-inhibitory domain(85). The catalytic domain has several binding sites for ATP and other substrate-anchored proteins [9]. It is responsible for transferring phosphate from ATP to Ser or Thr residues in the substrate. The autoinhibition domain is characterized by a pseudo-substrate site, which binds to the catalytic domain and blocks its ability to phosphorylate proteins. The structural feature that controls this autoinhibition is threonine 286 residue [10]. Phosphorylation at this site will permanently activate the CaMKII enzyme. Once the Threonine 286 residue is phosphorylated, the inhibitory domain is blocked from the pseudo-substrate site, allowing the CaMKII enzyme to be permanently activated. Even without calcium and calmodulin, CamKII can remain active. The CaATP_NAI motif of S is "APAICHDGKAHFPRE", which overlaps with the CaMKII_AD motif "AQEKNFTTAPAICHDGKAHFPREGVFVSNGTHW". Obviously, there is no threonine (L-Threonine) in the CaATP_NAI motif and the overlapped CaMKII_AD motif. Therefore, S2 protein may not depend on Threonine phosphorylation for permanent activation. The $\mathrm{S}$ protein CaATP_NAI has the function of the catalytic domains and auto-regulatory domain of the CAMKII protein.

\subsection{Related domains of CaMKII_like system activation}

Table 2 shows that the motifs of DUF4440, EF-hand, Protein kinase, and SnoaL-like domains of S protein are all shorter and have a common motif “WPWYIW” (1212-1217). In those domains, the consensus motif is the C-terminal CaMKII_AD domain (1202-1256), outside the viral transmembrane domain. The highly conserved 8-residue sequence (KWPWY/WVWL) is necessary for membrane fusion but not crucial for $\mathrm{S}$ protein incorporation into particles(78). The specific motif also mediates the particle assembly of coronavirus spikes(78). It mainly related the functions of these domains to the activation of the CaMKII_AD domain function. These domains may be similar to $\mathrm{Ca}^{2+} / \mathrm{CaM}$ binding region.

SnoaL-like domain. It is a polyketide cyclase biosynthesized by Nogalamycin, which uses a twisted alpha-beta barrel fold(86). SnoaL-like catalyzes the loop-closing step in the biosynthesis of polyketide antibiotics produced by Streptomyces. SnoaL-like plays an important role in the binding of aglycones. The SnoaL-like domain motifs of $\mathrm{S}$ are "WPWYIW" and "YHKNNKSWMES". The "WPWYIW" motif is in the S2 protein. The "YHKNNKSWMES" motif is in the S1 protein. The motif "YHKNNKSWMES" is also in the N-terminal CaMKII_AD domain (136-158). It represents that SnoaL-like domain is related to CaMKII_AD function.

EF-hand domain. It is a helix-loop-helix domain or structural motif(87) found in calcium-binding proteins. $\mathrm{Ca}^{2+}$ ions and their ligands are in the loop. The EF-hand contains two alpha helices connected by a short loop region, commonly used to bind calcium ions. EF-hand has a high selectivity for calcium(88). It also finds EF-hand in calmodulin(89) and the muscle protein 
troponin C. After binding to $\mathrm{Ca}^{2+}$, the motif changes its conformational. Calmodulin realizes the function of $\mathrm{Ca}^{2+}$ regulation, such as the $\mathrm{Ca}^{2+}$ effector. The calmodulin molecule is a highly conserved single-chain polypeptide composed of 148 amino acid residues, comprising two domains at the N-terminal and $\mathrm{C}$-terminal, and a spiral structure connected in the middle. Each domain consists of two EF-hand motifs(90), and each calmodulin molecule contains $4 \mathrm{Ca}^{2+}$ binding sites. Calmodulin is in an open state only after combining with calcium ions and has biological activity. Therefore, calmodulin can detect the cytosolic calcium ions level. The functions of EF-hand may be very extensive. The semi-continuous $\mathrm{Ca}^{2+}$ binding site in D-galactose binding protein contains a loop of nine residues. Seven protein oxygen atoms, five of which are from the ring ( coordinated the $\mathrm{Ca}^{2+}$ ion), mimic the canonical EF ring $(91)$. Beside, Glu. Wingo et al. proposes a new mechanism to regulate the function of $\mathrm{Na}$ channels, in which $\mathrm{Ca}^{2+}$ ions directly bind to hH1 through the EF-hand matrix of the C-terminal domain(92). EF-hand motif of S protein is "YEQYIKWPWYIWLGF". We believed the region of EF-hand motif bound to $\mathrm{Ca}^{2+}$, had a calmodulin-like role and promoted the $\mathrm{S}$ conformation change. It is also interesting that ORF3a, ORF6, ORF7b, ORF10, and some orflab proteins also have EF-hand motif and may also have similar functions to calmodulin.

DUF4440 domain. The domain superfamily (IPR032710) similar to NTF2 (149). The NTF2 protein has a beta-alpha(2)-beta insertion behind the primary helix. This nuclear envelope protein promotes protein transport to the nucleus. The protein of this domain also includes calcium/calmodulin-dependent protein kinase II, the association domain (IPR013543). The $\mathrm{S}$ protein DUF4440 motif "QYIKWPWYIW" may mainly play the role of CaMKII_AD, assisting with the formation of HR1-HR2 six-helix bundles.

Protein kinase domain. PK_Tyr_Ser-Thr represents the catalytic domain(93) found in much serine/threonine and tyrosine protein kinases, excluding the catalytic domain of dual-specificity kinases. PK_Tyr_Ser-Thr is responsible for the reversible process mediated by protein kinase and phosphoprotein phosphatase. Phosphorylation usually changes the target protein's function by changing the activity of the enzyme, the location of the cell, or the association with other proteins. We detected that S protein did not have PK Tyr_Ser-The domain, but have a protein kinase domain ("YEQYIKWPWYIW").

Protein kinases play a role in many cellular processes, including division, proliferation, apoptosis, and differentiation(94). Protein kinases catalyze the transfer of gamma-phosphate from a nucleotide triphosphate (usually ATP) to one or more amino acid residues in the side chain of a protein substrate. It makes conformational changes that affect protein function(93). The phosphorylation by the protein kinase domain is related to the conformational change of S protein. The phosphorylation sites identified on the $\mathrm{S}$ glycoprotein are important in the trimer's assembly(47). The residues T29, S31, S349, T791, and S816 of the S protein are all on the surface, while T240 is located under the disordered ring. When the ring is phosphorylated, it will add a negative charge, affecting the ring conformation. Residues Y789 and T791 are at the interface of subunits and may participate in the control of trimer assembly. S637 and S640 are in a model ring; they are arranged together in order, and are nicely folded into these models in the ring modeled for compact folding, such as hairpins. In chains with extended domains, the same cyclic model has a comprehensive form. It will be consistent with phosphorylation that forces the hairpins apart at S637 and S640. 


\subsection{The CaATP_NAI domain was the membrane fusion motor of the S2 protein of SARS-COV-2}

For SARS-COV S and MERS S protein, we adopted the same domain search method of SARS-COV-2 S protein. Since many motif fragments were not conducive to analysis, we merged the motif sequences of the search results. The relevant structural domains of the CaMKII_Like system of SARS-COV are shown in Table 3, and the relevant structural domains of the CaMKII_Like system of MERS are shown in Table 4. It can be seen from the two tables that SARS-COV, MERS also have CaATP_NAI and CaMKII_AD domains, but their motifs are different from SARS-COV-2. SARS-COV's motif "RVDFCGKGYHLMSFPQAAPH " (CaMKII_AD) is similar to the SARS-COV-2's motif "RVDFCGKGYHLMSFPQSAPH" (CaMKII_AD). SARS-COV-2's motif "ELGKYEQYIKWPWYIWLGFIAGLIAIVMVTIMLCCMTSCCSCLKGCCSCGSCCKF"

(CaMKII_AD) is origined from “NLNESLIDLRELGKYEQYIKWPWYVWLGFIAGLIAIVMVT” (CaMKII_AD) and MERS's motif “CCTGCGTNCMGKLKCNRCCDRYEEYDLEPHKVHVH” (CaMKII_AD). This SARS-COV motif is at the N-terminal, and this MERS motif is at the C-terminal. Both CaATP_NAI and CaMKII_AD are also overlap. But, the CaATP_NAI motifs of the three species are different, which may be a significant cause of SARS-COV-2 specific fusion events. Beside, DUF4440, EF-hand, Protein kinase, and SnoaL-like domains all include the "WPWY[I/V]W" motif. The position and function of this motif are similar, indicating that the site is high conservative.

We used the local version of ClustalX2 to perform multiple sequence alignments of the $\mathrm{S}$ proteins of the three species. Based on this comparison result, we employed the online ENDscript server(95) to plot the comparison result and marked the EF-hand and CaATP_NAI domains on the result map (Figure 1). Since the original image is huge, we only intercepted the sequence near the EF-hand and CaATP_NAI domains. The comparison result of S proteins shows that SARS-COV-2 and SARS protein are very similar, with more point mutation differences. However, MERS and SARS-COV-2 (or SARS) are pretty different. In addition to some mutations, there are many insertion mutations. The three species have a similar EF-hand domain sequence "WPWY[I/V]WLGF". The motif "KWPWY/WVWL" has been confirmed by experiments reported in the literature to participate in membrane fusion(78).

Table 3. CaMKII-like related motifs possessed by S protein of SARS-COV

\begin{tabular}{llrr}
\hline \multicolumn{1}{c}{ Domain } & \multicolumn{1}{c}{ Motif } & Start & End \\
\hline CaATP_NAI & YRYLRHGKLRPFERDI & 440 & 455 \\
CaMKII_AD & YNYKYRYLRHGKLRPFERDISNVP & 436 & 459 \\
& NLNESLIDLRELGKYEQYIKWPWYVWLGFIAGLIAIVMVT & 1174 & 1213 \\
& HEGKAYFPR & 1065 & 1073 \\
& RVDFCGKGYHLMSFPQAAPH & 1021 & 1040 \\
& TKMSECVLGQSKRVDFC & 1009 & 1025 \\
DUF4440 & QYIKWPWYVW & 1190 & 1199 \\
EF-hand & DDFMGCVLAWNTRNIDATSTGNYNYKYRYLRHGK & 414 & 447 \\
& WPWYVWLGF & 1194 & 1202 \\
Protein kinase & GKYEQYIKWPWYVW & 1186 & 1199 \\
SnoaL-like & RELGKYEQYIKWPWYVW & 1183 & 1199 \\
\hline
\end{tabular}


Table 4. CaMKII-like related motifs possessed by S protein of MERS

\begin{tabular}{clrr}
\hline \multicolumn{1}{c}{ Domain } & \multicolumn{1}{c}{ Motif } & Start & End \\
\hline CaATP_NAI & CCTGCGTNCMGKLKCNRC & 1319 & 1336 \\
CaMKII_AD & CCTGCGTNCMGKLKCNRCCDRYEEYDLEPHKVHVH & 1319 & 1353 \\
& CTFMYTYNITEDEILEWF & 237 & 254 \\
& KELGNYTYYNKWPWYIWLGFIAG & 1284 & 1306 \\
& PLEGGGWLVASGSTVAMTEQLQMGF & 547 & 571 \\
& PNGLYFMHVGYYPSNHIE & 1131 & 1148 \\
DUF4440 & KWPWYIW & 1294 & 1300 \\
EF-hand & KLKCNRCCDRYEEYDLEPHKVHVH & 1330 & 1353 \\
& WPWYIWLGF & 1295 & 1303 \\
Protein kinase & NCMGKLKCNRCCDRYEEYDLEPHKVHVH & 1326 & 1353 \\
& KWPWYIW & 1294 & 1300 \\
& RCCDRYEEYDLEPHKVHVH & 1335 & 1353 \\
& KWPWYIW & 1294 & 1300 \\
\hline
\end{tabular}

The positions of the CaATP_NAI domains of the three species are quite different. The CaATP_NAI of MERS is located at the end of C-terminal. The CaATP_NAI of SARS-COV-2 is located at the C-terminal (upstream of MERS), and the CaATP_NAI of SARS-COV is located at the N-terminal. The difference in MERS is caused by insertion mutations and point mutations. The difference in SARS-COV is caused by point mutations. The difference in position reflects the different roles of CaATP_NAI in the three species. The CaATP_NAI of SARS-COV is located in the $\mathrm{S} 1$ protein. It may be mainly responsible for participating in receptor binding and may contribute less to the fusion of S2 protein. The CaATP_NAI of MERS is at the C-terminus of S2 protein, close to the cytoplasmic region of the virus. The fragment near the $\mathrm{C}$-terminus is also the CaMKII_AD region of SARS-COV-2. So, CaATP_NAI of MERS may be responsible for driving S2 conformational changes, and less contribute to the fusion too. The CaATP_NAI of SARS-COV-2 is at the C-terminal of the S2 protein, outside the virus membrane, and at the interface between the virus and the cell. An ATP motor at this position will undoubtedly give the S2 protein of SARS-COV-2 a strong driving force to achieve precise membrane positioning and fusion. However, the S2 proteins of SARS-COV and MERS lack this motor. Therefore, cell infections of SARS-COV and MERS are often manifested in the form of endocytosis. SARS-COV-2 cell infections are not only in the formation of endocytosis but also in membrane fusion. 


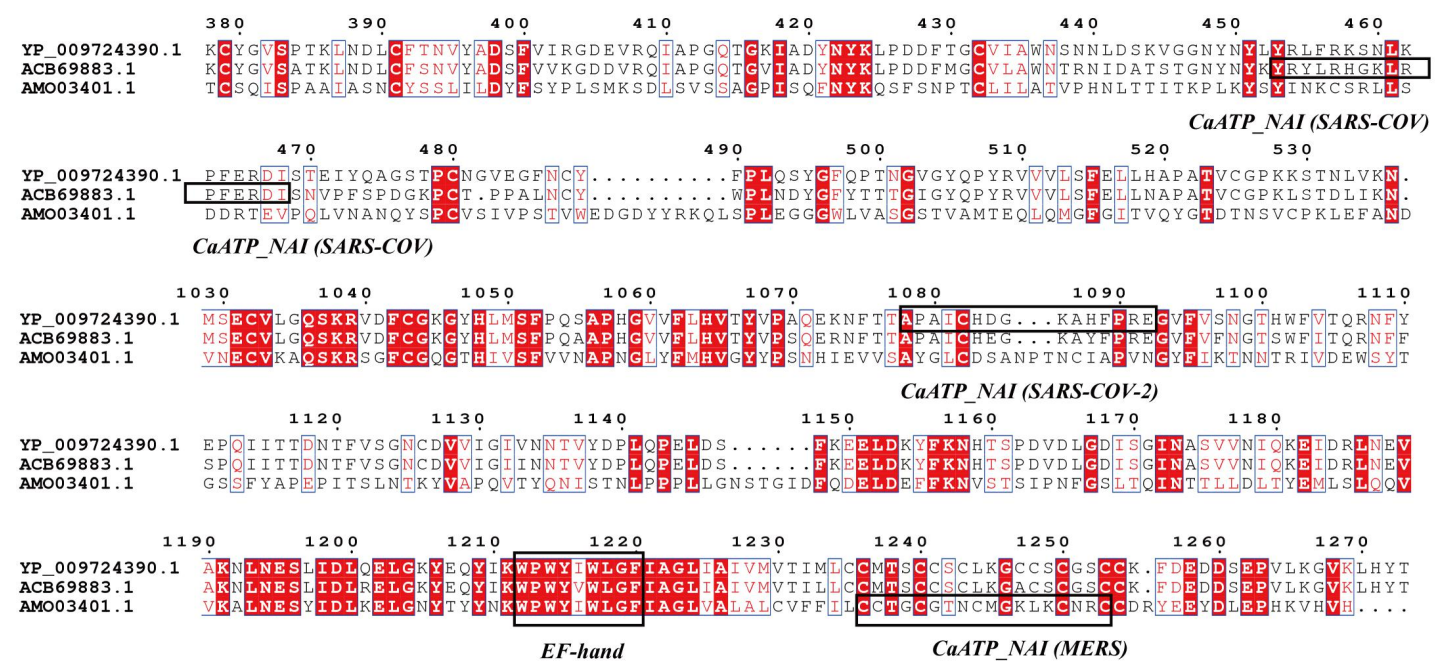

Figure 1. CaATP_NAI domains of S protein between SARS-COV-2, SARS, and MERS. YP_009724390.1 is the S protein of SARS-COV-2. ACB69883.1 is the S protein of SARS-COV. AMO03401.1 is the $S$ protein of MERS.

\subsection{CaMKII_Like system mediated the fusion of viral membrane and cell membrane}

The crystal structure shows that the linker region upstream of the HR2 motif experienced a sizeable structural rearrangement during membrane fusion(74). Its correct refolding is vital for the formation of the HR1-HR2 six-helix bundle. HR1-HR2 six-helix bundles play an essential role in membrane fusion. The correct refolding of the linker region brings the viral membrane close to the host membrane. Before fusion, the $\beta$-strand of the linker region and the SD3 domain form a $\beta$-sheet. After fusion, the linker area is rearranged into a groove-shaped extension structure mainly assembled from rings joined along the axis of the ring. Short helices surround the distal membrane end of the central helix bundle and $\beta$-sheets. The upstream helix(UH), $\beta$-hairpin(BH) motif, and SD3 motif are located around the C-terminus of the central HR1-CH helix. The SD3 motif part covers the top of the central spiral area. Unlike the S1 subunit, the S2 subunit shows low variability(72). The HR domain and the interaction between HR1 and HR2 are also highly conserved(73). Two clinically approved drugs, itraconazole(ITZ) and estradiol benzoate (EB), have been found to target the Six-helix(6-HB) fusion core to inhibit virus entry(44).

We downloaded the SARS-COV-2 S open state crystal structure 6vyb from the PDB database and then marked the main CaATP_NAI and CaMKII_AD domains on the structure (Figure 2). Figure 2 shows that the CaATP_NAI and CaMKII_AD domains are both near the outer membrane of the virus. The CaMKII_AD domain motif "MQMAYRF" is a helical structure, while the CaMKII_AD domain motifs "RVDFCGKGYHLMSFPQSAPH" and "AQEKNFTTAPAICHDGKAHFPREGVFVSNGTHW" are folded structures. However, the CaMKII_AD domain motif "ELGKYEQYIKWPWYIWLGFIAGLIAIVMVTIMLCCMTSCCSCLKGCCSCGSCCKF" is a transmembrane structure and is not in the 6vyb structure diagram.

There is no SARS-COV-2 HR1-HR2 hexamer in the PDB database. Because the structure of the HR1-HR2 hexamer is relatively conservative, we marked the position of the CaATP_NAI domain of SARS-COV-2 on the HR1-HR2 hexamer of SARS-COV (Figure 3). Figure 3 shows that CaATP_NAI and CaMKII_AD overlap so that the folded sheet is near the outer membrane of 
the virus. There is also a conservative CaMKII_AD domain motif "RVDFCGKGYHLMSFPQSAPH" near CaATP_NAI. The motif of SARS-COV here is "RVDFCGKGYHLMSFPQAAPH". CaATP_NAI may drive the HR1-HR2 hexamer to fold irreversibly toward the cell membrane to promote the proximity of the virus membrane to the cell membrane. The CaATP_NAI position difference between SARS-COV-2 and SARS-COV is similar to the rear-wheel drive and front-wheel drive of a car.

In vesicle fusion, SNAREs interact with vesicle tethers in a calcium-dependent manner(96). In the presence of $\mathrm{Ca}^{2+}$, t-SNARE, and v-SNARE in the opposite bilayer interact. They self-assemble into a ring conformation to form conductive and leak-proof channel--- $\mathrm{Ca}^{2+}$ bridges. Double-layer calcium bridging tightly binds bilayers. It releases water from hydrated $\mathrm{Ca}^{2+}$ ions and displaces loosely coordinated water on the phosphate groups in the lipid membrane(97). Therefore, it discharges water at the contact site between the bilayers, causing membrane instability: lipid mixing and membrane fusion. t-/v-SNARE is a tight complex, and its disassembly requires an NSF-ATPase, which acts as a right-hand molecule for movement(98). We speculated that SARS-COV-2 membrane fusion was also calcium-induced and a similar calcium bridge was formed.

After S1/S2 cleavage sites are cut, the EF-hand domain of S protein bound to calcium ions, $\mathrm{S} 2$ protein had CaMKII protein activities. It inserted the fusion peptide of the S2 protein into the cell membrane, fixing the S2 protein on the cell membrane. Then the conformation of the CaMKII_AD domains changed and drove S2 protein to form HR1-HR2 six-helix bundles. The HR1-HR2 hexamer could be folded toward the viral membrane. The HR1-HR2 hexamer near the viral membrane (contact position) had three self-inhibition domains---CaATP_NAI domain, an CaATPases enzyme activated by $\mathrm{Mg} 2+$ and releases energy through ATP phosphorylation, and was also a special $\mathrm{Ca}^{2+}$ pump. The CaATP_NAI domain could be anchored on the cell (or viral) membrane and move on the cell (or viral) membrane. The three CaATP_NAI domains moved in a specific rotation direction, driving the HR1-HR2 hexamer to fold irreversibly toward the viral membrane. This folding movement brought the virus membrane closer to the cell membrane.

When the viral membrane is very close to the cell membrane, the CaATP_NAI and CaMKII_AD domain at the contact site of the HR1-HR2 hexamer extended outward to form a groove-shaped barrel structure. The barrel was connected with the virus membrane and the cell membrane simultaneously. $\mathrm{Ca}^{2+}$ was gathered in the barrel structure to combine with water molecules to form hydrated $\mathrm{Ca}^{2+}$ ions, creating a calcium bridge between the contact surfaces of the two membranes. It released water from the hydrated $\mathrm{Ca}^{2+}$ ions and displaced loosely coordinated water from the phosphate groups of the lipid membrane. The contact between the bilayer membranes discharged water, causing membrane instability, triggering lipid mixing and fusion of the membrane lobules. The CaATP_NAI drove the conformational change of the contact position of the HR1-HR2 hexamer, disassembling the grooved barrel structure. The scale of fusion between the virus membrane and the cell membrane was expanded. The original two membrane contact surfaces formed a channel connecting the virus and the cytoplasm. The HR1-HR2 hexamer fell into the cytoplasm and was captured and decomposed by the proteolytic system. Eventually, the virus's membrane and membrane proteins were fused to the cell membrane. The virus's nucleocapsid and RNA complexes were injected into the infected cytoplasm.

The S protein of the infected cell may bind to the ACE2 receptor of another cell (or also an infected cell) and then achieve membrane fusion through a similar principle, forming cell syncytia, 
includes syncytial multinucleated giant cells.

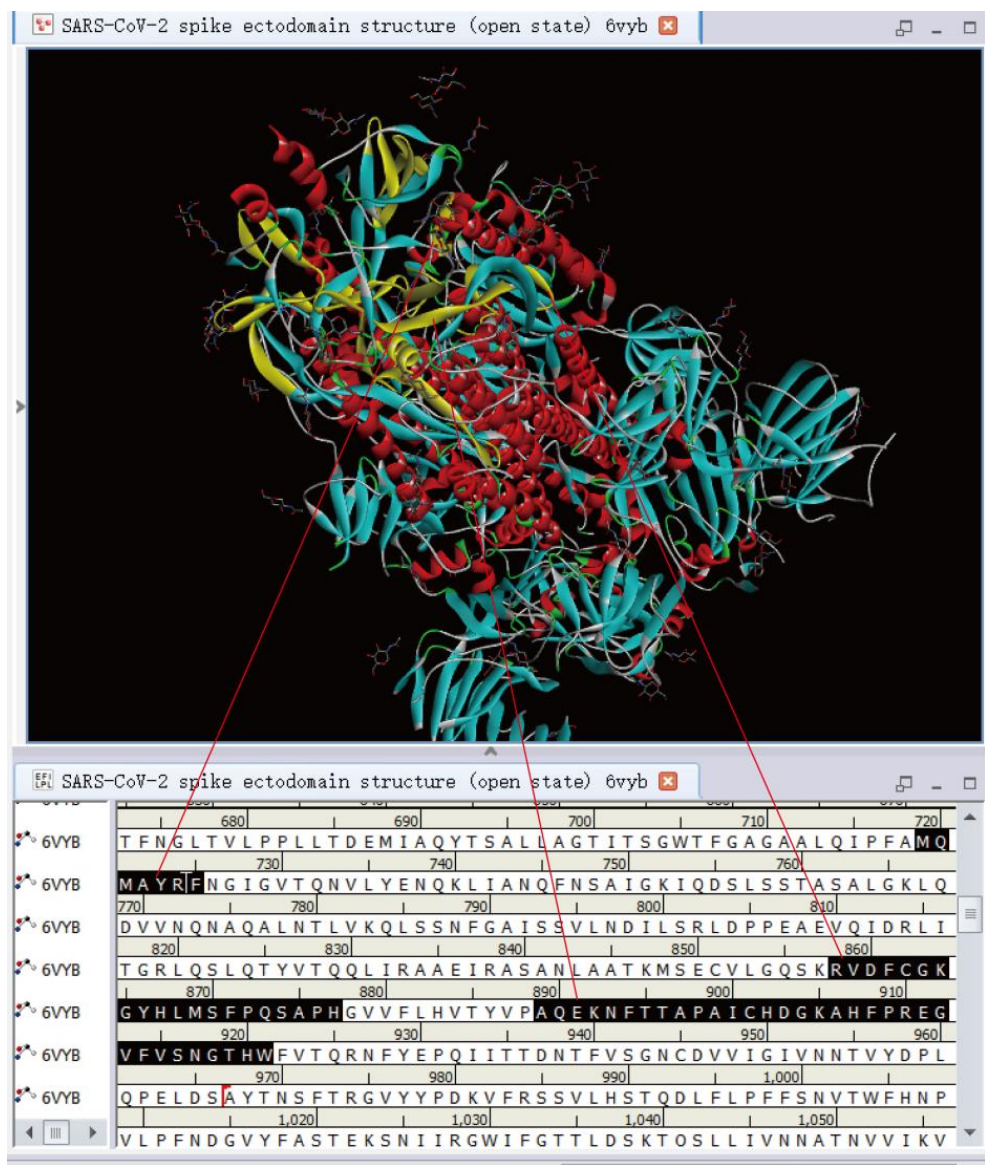

Figure 2. The position of some CaATP_NAI and CaMKII_AD domains of SARS-COV-2 S (PDBID: 6vyb). The bottom right corner of the picture belongs to the S1 protein, close to the cell. The upper left corner of the picture belongs to the S2 protein, close to the viral membrane.

A

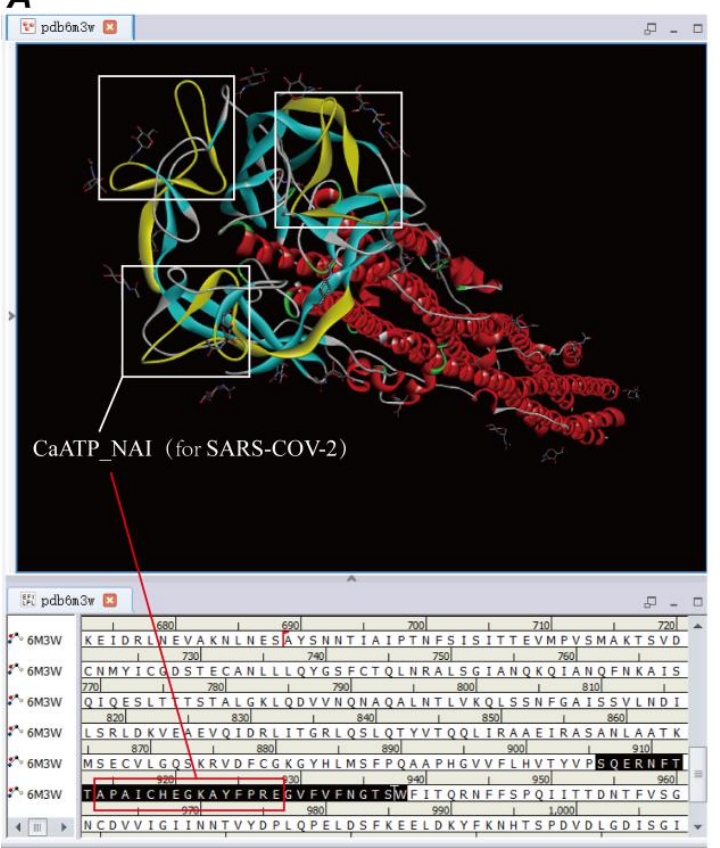

B

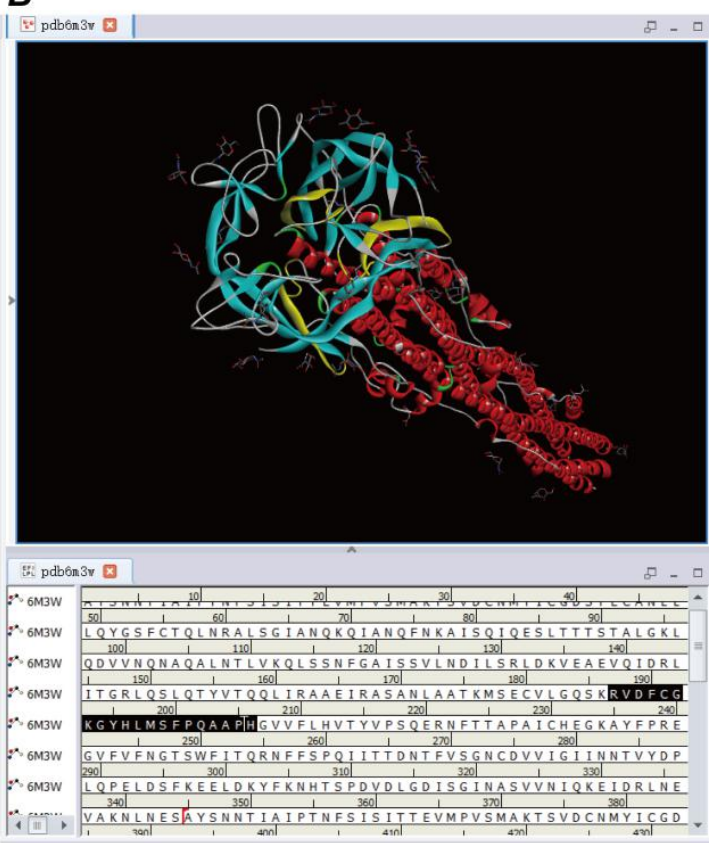


Figure 3. The position of the CaATP_NAI of SARS-COV-2 S in the HR1-HR2 hexamer (PDBID: 6m3x). R1-HR2 hexamer belongs to S2 protein. There is no S1 protein in the lower right corner of the picture, close to the cell membrane. The upper left corner of the image is near the viral membrane. $6 \mathrm{~m} 3 \mathrm{x}$ is the SARS-COV crystal structure, and there is no SARS-COV-2 HR1-HR2 hexamer in the PDB database. The HR1-HR2 hexamer structure of SARS-COV-2 and SARS-COV are similar. $\boldsymbol{A}$. The CaATP_NAI motif of SARS-COV-2 (three white boxes in the figure) is "APAICHDGKAHFPRE", which overlaps with the CaMKII_AD domain there. There are point mutations in the sequences between SARS-COV-2 and SARS-COV. Therefore, SARS-COV does not form a CaATP_NAI domain in the white box. B. Both SARS-COV-2 and SARS-COV have a conserved CaMKII_AD domain "RVDFCGKGYHLMSFPQ[S/A]APH", which is close to CaATP_NAI.

\section{Discussion}

\subsection{The increased calcium demand during membrane fusion could disrupt the calcium homeostasis}

The protein-bound calcium in plasma is called non-diffusible calcium and cannot penetrate the capillary wall. Ionized calcium(99) and Calcium compounds such as calcium citratein plasma(100) are called diffusible calcium, which can penetrate the capillary wall(101). In plasma calcium, only ionized calcium directly plays a physiological role, and calcium ions play an essential role in the excitability and contraction of vascular smooth muscle. Non-diffusible calcium in plasma has no direct physiological effect, and is in a dynamic balance with ionized calcium, and is affected by blood $\mathrm{pH}$. When the blood $\mathrm{pH}$ decreases, it promotes the dissociation of bound calcium and increases $\mathrm{Ca}^{2+}$; conversely, when the $\mathrm{pH}$ increases, the bound calcium increases, and $\mathrm{Ca}^{2+}$ decreases.

Vitamin D (calcosterol) is combined in active form with calcitonin from thyroid C cells and parathyroid hormone (PTH) from parathyroid glands. It keeps circulating calcium levels in the body within a narrow concentration range(102). The increase in plasma calcium is because of the mobilization of calcium in the bones, the increase in calcium absorption, and the stimulation of vitamin D3 (calcitriol) synthesis by the kidneys. Calcitriol increases calcium absorption from the intestines and works synergistically with PTH to absorb calcium from the bones. Together, these hormone-mediated events normalize serum calcium levels.

This study found that the CaMKII-like domains of the S protein mediated the fusion of virus and infected cells, and it involved calcium ions in this process. In membrane fusion, calcium ions near the interface between S protein and cell membrane quickly accumulated, which produced abnormal calcium ion currents. Abnormal flow of calcium ions caused depolarization of cells near the infected cell, prompting many calcium ions to flushed into nearby cells. Finally, the calcium ion demand in serum accelerated in a short time. It promoted the release of calcium in the body. If the SARS-COV-2 virus infection area was large, it encouraged the abnormality of serum calcium levels and disrupted the body's calcium homeostasis.

\subsection{Membrane fusion increased the risk of coagulation and vascular calcification}

The coagulation of COVID-19 patients may have many factors. It is not clear whether clotting or hemolysis must occur during membrane fusion between the SARS-COV-2 virus and 
the infected cell. In membrane fusion, the cytoplasmic domain of the $\mathrm{S}$ protein and the surface CAF-motif (Cysteine Aggregation Fusion) of the infected cell may assist the formation of blood clots(78). This study found that the S protein has a CaMKII_Like conserved domain. There is an EF-hand domain "YEQYIKWPWYIWLGF" upstream of CAF-motif, that is, in the N-terminal direction, a region that binds calcium ions. CAF-motif belongs to the C-terminal of the CaMKII_AD("ELGKYEQYIKWPWYIWLGFIAGLIAIVMVTIMLCCMTSCCSCLKGCCSCGS CCKF") domain, and EF-hand belongs N-terminal of the CaMKII_AD domainto. The CaMKII_AD at C-terminal of S protein, promoting the formation of the HR1-HR2 hexamer involved in the fusion process. The CaMKII-like system of S protein induced the fusion of virus and infected cells. Many calcium ions were consumed during the fusion process. The membrane fusion process caused many calcium ions near the interface between the virus and the infected cell to accumulate in a short time. Calcium ion was a coagulation factor, so that clots may occur near the contact surface. SARS-CoV-2 infection depends on cell heparan sulfate(103) and ACE2. Heparan sulfate and dermatan sulfate have anticoagulant effects(104). If lower levels of heparan sulfate cannot neutralize coagulation factors in infection and fusion, it will risk clotting. Therefore, if cytokines and oxidative stress factors were not considered, abnormal calcium release and collect mainly induced the coagulation reaction of COVID-19 patients because of a viral infection and membrane fusion. The heme theory believed that the SARS-COV-2 virus could attack the hemoglobin in red blood cells(105). The SARS-COV-2 virus could infect red blood cells through the $\mathrm{CD} 147$ receptor. Calcium ions triggered coagulation during the membrane fusion process. Therefore, hemolysis in membrane fusion was an infrequent phenomenon.

In addition, it attached calcium to the endothelium of blood vessels easily to form atherosclerotic plaques. The systemic inflammatory response causes by acute infection intensified the inflammatory activity in the coronary atherosclerotic plaque, making it prone to rupture and causing acute coronary syndrome(106). Vascular calcification was the pathological basis of cardiovascular disease. It correlated vascular calcification with the severity of cardiovascular disease. Intimal calcification was the most dangerous. Atherosclerotic calcification easily increased blood pressure, heart failure, myocardial ischemia, and even myocardial infarction.

\section{Conclusion}

COVID-19 is a unique disease characterized by extensive pulmonary thrombosis, and the formation of infected cell syncytia, includes syncytial multinucleated giant cells. The syncytial cells is relating to extensive tissue damage. Studies show that SARS-CoV-2 S protein mediates lymphocyte elimination through syncytia. Calcium triggers protease-mediated membrane fusion activation of SARS-COV-2. The SARS-CoV-2 S protein on the membrane of infected cells can initiate receptor-dependent syncytia formation. However, the mechanism by which the SARS-CoV-2 S protein regulates the construction of these syncytia is not fully clear. Therefore, it is of great significance to study how the SARS-CoV-2 S protein drives membrane fusion and induces syncytia.

This study adopted structural domain search methods to analyze the structural and non-structural proteins of the SARS-COV-2 virus. The results showed that the surface glycoprotein (S) had conserved domains of CaMKII: CaMKII_AD, CaATP_NAI, DUF4440, EF-hand, Protein kinase, and SnoaL-like. These conserved domains were mainly in the S2 protein and nearby regions. CaATP_NAI and CaMKII_AD are highly overlapped and located upstream of 
the C-terminal of S2. But, the CaATP_NAI of SARS-COV is located at the N-terminal, on S1 protein. The CaATP_NAI of MERS is located at the end of the C-terminal, near the inner of the viral membrane. The CaATP_NAI of SARS-COV-2 is located at the C-terminal (upstream of MERS), close to the outer of the viral membrane. DUF4440, EF-hand, Protein kinase, and SnoaL-like domains are all shorter and have a highly conservative motif "WPWYIW". EF-hand motif, "YEQYIKWPWYIWLGF", is the calcium binding domain. There is also a highly conserved $\mathrm{AD}$ domain near the C-terminus of $\mathrm{S}$, which contains the "WPWYIW" motif and CAF-motif. It may play an essential role in forming the HR1-HR2 hexamer conformation.

We believed that after the EF-hand domain of S protein bound to calcium ions, S2 protein had CaMKII protein activities. After the S protein fusion peptide was inserted into the infected cell membrane and fixed the S2 protein on the cell membrane, the CaMKII_AD domain changed S conformation, prompting the S2 protein to form HR1-HR2 six-helix bundles. The HR1-HR2 hexamer had three CaATP_NAI domains ("APAICHDGKAHFPRE") near the viral membrane (contact position), an enzyme activated by $\mathrm{Mg} 2+$, and released energy through ATP phosphorylation. The CaATP_NAI domain could anchor and move on the cell(or viral) membrane. The CaATPase drove the HR1-HR2 hexamer on contact position, changed the conformation, and folded irreversibly toward the viral membrane. The CaATP_NAI and CaMKII_AD domain extended to the outside and combined the viral membrane and the cell membrane so that the contact position of the two membranes formed a thin barrel structure. The hydrated calcium ions gathered in the barrel structure to create a calcium bridge. The hydrated calcium ions released water outward, and loosely coordinated water on the phosphate groups of the lipid membrane also was replaced. The release action of water caused the instability of the double membrane, triggering lipid mixing and fusion of the membrane. CaATPase drove the conformational change of the HR1-HR2 hexamer, disassembled the barrel structure, and then HR1-HR2 hexamer fell into cytoplasm. Finally, the viral membrane fused with the cell membrane on a large scale. The virus contents mixed with the cytoplasmic of the cell. The S protein of the infected cell could bind to the ACE2 receptor of another cell (or also an infected cell) and then achieve membrane fusion through a similar principle. It formed cell syncytia, includes syncytial multinucleated giant cells, by the manner.

It undoubtedly that CaATPase gave the S2 protein of SARS-COV-2 a strong driving force to achieve precise membrane positioning and fusion, forming the infected cell syncytia such as syncytial multinucleated giant cells. However, SARS-COV and MERS lacked the motor at the position.

\section{Declarations}

\section{Ethics approval and consent to participate}

Not applicable.

\section{Consent for publication}

Not applicable.

\section{Availability of data and material}

The datasets and results supporting the conclusions of this article are available at : https://pan.baidu.com/s/1OBS_AKjMZGWJhPfS5T_hXQ; code: 5kvg 
Or:https://mega.nz/folder/12phVagY\#z8vweprF7d8dMbnsJlYZ2g

\section{Competing interests}

The authors declare that they have no competing interests.

\section{Funding}

This work was funded by a grant from the National Natural Science Foundation for the Talent Introduction Project of Sichuan University of Science and Engineering (award number: 2018RCL20, grant recipient: WZL).

\section{Author's contribution}

Funding was obtained by WZL. Besides, design, analysis and writing are finished by WZL, while data curation and manuscript check are undertaken by HLL. Both authors have read and agreed to the published version of the manuscript.

\section{Acknowledgements}

Not applicable.

\section{Author details}

${ }^{1}$ School of Computer Science and Engineering, Sichuan University of Science \& Engineering, Zigong, 643002, China.

${ }^{2}$ School of Life Science and Food Engineering, Yibin University, Yibin, 644000, China.

\section{References}

1. R. Bussani, E. Schneider, L. Zentilin, C. Collesi, H. Ali, L. Braga, M. C. Volpe, A. Colliva, F. Zanconati, G. Berlot, F. Silvestri, S. Zacchigna, M. Giacca, Persistence of viral RNA, pneumocyte syncytia and thrombosis are hallmarks of advanced COVID-19 pathology. EBioMedicine 61, 103104 (2020); published online Epub2020/11/01/ (https://doi.org/10.1016/j.ebiom.2020.103104).

2. M. F. Santana, R. A. d. A. Pinto, B. H. Marcon, L. C. A. S. d. Medeiros, T. B. d. N. d. Morais, L. C. Dias, L. P. d. Souza, G. C. d. Melo, W. M. Monteiro, M. V. G. Lacerda, Pathological findings and morphologic correlation of the lungs of autopsied patients with SARS-CoV-2 infection in the Brazilian Amazon using transmission electron microscopy. Revista da Sociedade Brasileira de Medicina Tropical 54, (2021).

3. A.-A. Keresztesi, F. Perde, A. Ghita-Nanu, C.-C. Radu, M. Negrea, G. Keresztesi, Post-Mortem Diagnosis and Autopsy Findings in SARS-CoV-2 Infection: Forensic Case Series. Diagnostics 10, 1070 (2020).

4. F. Calabrese, F. Pezzuto, F. Fortarezza, P. Hofman, I. Kern, A. Panizo, J. von der Thüsen, S. Timofeev, G. Gorkiewicz, F. Lunardi, Pulmonary pathology and COVID-19: lessons from autopsy. The experience of European Pulmonary Pathologists. Virchows archiv, 1-14 (2020).

5. Z. Xu, L. Shi, Y. Wang, J. Zhang, L. Huang, C. Zhang, S. Liu, P. Zhao, H. Liu, L. Zhu, Pathological findings of COVID-19 associated with acute respiratory distress syndrome. The Lancet respiratory medicine 8, 420-422 (2020).

6. C.-H. Chen, S. Badeti, J. H. Cho, A. Naghizadeh, X. Wang, D. Liu, Deletion of ER-retention Motif on SARS-CoV-2 Spike Protein Reduces Cell Hybrid During Cell-cell Fusion. Res Sq, rs.3.rs-380389 (2021)10.21203/rs.3.rs-380389/v1). 
7. Z. Zhang, Y. Zheng, Z. Niu, B. Zhang, C. Wang, X. Yao, H. Peng, D. N. Franca, Y. Wang, Y. Zhu, Y. Su, M. Tang, X. Jiang, H. Ren, M. He, Y. Wang, L. Gao, P. Zhao, H. Shi, Z. Chen, X. Wang, M. Piacentini, X. Bian, G. Melino, L. Liu, H. Huang, Q. Sun, SARS-CoV-2 spike protein dictates syncytium-mediated lymphocyte elimination. Cell Death \& Differentiation, (2021); published online Epub2021/04/20 (10.1038/s41418-021-00782-3).

8. S. A. Theuerkauf, A. Michels, V. Riechert, T. J. Maier, E. Flory, K. Cichutek, C. J. Buchholz, Quantitative assays reveal cell fusion at minimal levels of SARS-CoV-2 spike protein and fusion from without. iScience 24, 102170 (2021); published online Epub2021/03/19/ (https://doi.org/10.1016/j.isci.2021.102170).

9. A. Puzyrenko, J. C. Felix, Y. Sun, H. Rui, Y. Sheinin, Acute SARS-CoV-2 pneumonitis with cytotoxic CD8 positive T-lymphocytes: Case report and review of the literature. Pathol Res Pract 220, 153380-153380 (2021)10.1016/j.prp.2021.153380).

10. S. Tian, Y. Xiong, H. Liu, L. Niu, J. Guo, M. Liao, S.-Y. Xiao, Pathological study of the 2019 novel coronavirus disease (COVID-19) through postmortem core biopsies. Modern Pathology 33, 1007-1014 (2020); published online Epub2020/06/01 (10.1038/s41379-020-0536-x).

11. M. Remmelink, R. De Mendonça, N. D’Haene, S. De Clercq, C. Verocq, L. Lebrun, P. Lavis, M.-L. Racu, A.-L. Trépant, C. Maris, S. Rorive, J.-C. Goffard, O. De Witte, L. Peluso, J.-L. Vincent, C. Decaestecker, F. S. Taccone, I. Salmon, Unspecific post-mortem findings despite multiorgan viral spread in COVID-19 patients. Critical Care 24, 495 (2020); published online Epub2020/08/12 (10.1186/s13054-020-03218-5).

12. S. Stadlmann, R. Hein-Kuhnt, G. Singer, Viropathic multinuclear syncytial giant cells in bronchial fluid from a patient with COVID-19. Journal of Clinical Pathology 73, 607-608 (2020).

13. V. J. Munster, F. Feldmann, B. N. Williamson, N. Van Doremalen, L. Pérez-Pérez, J. Schulz, K. Meade-White, A. Okumura, J. Callison, B. Brumbaugh, Respiratory disease in rhesus macaques inoculated with SARS-CoV-2. Nature 585, 268-272 (2020).

14. B. Rockx, T. Kuiken, S. Herfst, T. Bestebroer, M. M. Lamers, B. B. O. Munnink, D. de Meulder, G. van Amerongen, J. van den Brand, N. M. Okba, Comparative pathogenesis of COVID-19, MERS, and SARS in a nonhuman primate model. Science 368, 1012-1015 (2020).

15. B. T. Bradley, H. Maioli, R. Johnston, I. Chaudhry, S. L. Fink, H. Xu, B. Najafian, G. Deutsch, J. M. Lacy, T. Williams, Histopathology and ultrastructural findings of fatal COVID-19 infections in Washington State: a case series. The Lancet 396, 320-332 (2020).

16. A. N. Duarte-Neto, E. G. Caldini, M. S. Gomes-Gouvêa, C. T. Kanamura, R. A. de Almeida Monteiro, J. F. Ferranti, A. M. C. Ventura, F. A. Regalio, D. M. Fiorenzano, M. A. B. C. Gibelli, W. B. d. Carvalho, G. N. Leal, J. R. R. Pinho, A. F. Delgado, M. Carneiro-Sampaio, T. Mauad, L. F. Ferraz da Silva, P. H. N. Saldiva, M. Dolhnikoff, An autopsy study of the spectrum of severe COVID-19 in children: From SARS to different phenotypes of MIS-C. EClinicalMedicine 35, 100850 (2021).

17. V. Canini, F. Bono, P. Calzavacca, G. Capitoli, G. Foti, F. Fraggetta, S. Galimberti, A. Gianatti, M. Giani, A. Nasr, G. Paciocco, F. Pagni, R. Rona, V. L'Imperio, Cytopathology of Bronchoalveolar Lavages in COVID-19 Pneumonia: A Pilot Study. Cancer Cytopathology n/a, https://doi.org/10.1002/cncy.22422).

18. C. J. Zeiss, S. Compton, R. T. Veenhuis, Animal Models of COVID-19. I. Comparative Virology and Disease Pathogenesis. ILAR J, ilab007 (2021) ). 
19. G.-C. Oprinca, L.-A. Muja, Postmortem examination of three SARS-CoV-2-positive autopsies including histopathologic and immunohistochemical analysis. International Journal of Legal Medicine 135, 329-339 (2021); published online Epub2021/01/01 (10.1007/s00414-020-02406-w).

20. G. Papa, D. L. Mallery, A. Albecka, L. G. Welch, J. Cattin-Ortolá, J. Luptak, D. Paul, H. T. McMahon, I. G. Goodfellow, A. Carter, S. Munro, L. C. James, Furin cleavage of SARS-CoV-2 Spike promotes but is not essential for infection and cell-cell fusion. PLOS Pathogens 17, e1009246 (2021)10.1371/journal.ppat.1009246).

21. S. Xia, M. Liu, C. Wang, W. Xu, Q. Lan, S. Feng, F. Qi, L. Bao, L. Du, S. Liu, C. Qin, F. Sun, Z. Shi, Y. Zhu, S. Jiang, L. Lu, Inhibition of SARS-CoV-2 (previously 2019-nCoV) infection by a highly potent pan-coronavirus fusion inhibitor targeting its spike protein that harbors a high capacity to mediate membrane fusion. Cell Research 30, 343-355 (2020); published online Epub2020/04/01 (10.1038/s41422-020-0305-x).

22. K. K. Stein, P. Primakoff, D. Myles, Sperm-egg fusion: events at the plasma membrane. Journal of Cell Science 117, 6269-6274 (2004)10.1242/jcs.01598).

23. L. K. Tamm, J. Crane, V. Kiessling, Membrane fusion: a structural perspective on the interplay of lipids and proteins. Current opinion in structural biology 13, 453-466 (2003); published online EpubAug (10.1016/s0959-440x(03)00107-6).

24. A. E. Smith, A. Helenius, How viruses enter animal cells. Science 304, 237-242 (2004); published online EpubApr 9 (10.1126/science.1094823).

25. R. Jahn, H. Grubmüller, Membrane fusion. Current opinion in cell biology 14, 488-495 (2002); published online EpubAug (10.1016/s0955-0674(02)00356-3).

26. Z. Daniloski, T. X. Jordan, H.-H. Wessels, D. A. Hoagland, S. Kasela, M. Legut, S. Maniatis, E. P. Mimitou, L. Lu, E. Geller, Identification of required host factors for SARS-CoV-2 infection in human cells. Cell 184, 92-105. e116 (2021).

27. A. Walls, Y. Park, M. Tortorici, A. Wall, A. McGuire, D. Veesler. (2020).

28. M. Hoffmann, H. Kleine-Weber, S. Pöhlmann, A multibasic cleavage site in the spike protein of SARS-CoV-2 is essential for infection of human lung cells. Molecular cell 78, 779-784. e775 (2020).

29. A. Z. Mykytyn, T. I. Breugem, S. Riesebosch, D. Schipper, P. B. van den Doel, R. J. Rottier, M. M. Lamers, B. L. Haagmans, SARS-CoV-2 entry into human airway organoids is serine protease-mediated and facilitated by the multibasic cleavage site. Elife 10, e64508 (2021).

30. M. Hoffmann, H. Kleine-Weber, S. Schroeder, N. Krüger, T. Herrler, S. Erichsen, T. Schiergens, G Herrler, N. Wu, A. Nitsche, SARS-CoV-2 cell entry depends on ACE2 and TMPRSS2 and is blocked by a clinically proven protease inhibitor. Cell. pii: S0092-8674 (20) 30229-4. Giet, D., Desmecht, D.(2020). A Covid-19-like syndrome associated with SARS-CoV-2 coronavirus in a domestic cat. Soumis pour publication, (2020).

31. M. Hoffmann, H. Kleine-Weber, N. Krüger, M. A. Mueller, C. Drosten, S. Pöhlmann, The novel coronavirus 2019 (2019-nCoV) uses the SARS-coronavirus receptor ACE2 and the cellular protease TMPRSS2 for entry into target cells. BioRxiv, (2020).

32. J. Buchrieser, J. Dufloo, M. Hubert, B. Monel, D. Planas, M. M. Rajah, C. Planchais, F. Porrot, F. Guivel-Benhassine, S. Van der Werf, N. Casartelli, H. Mouquet, T. Bruel, O. Schwartz, Syncytia formation by SARS-CoV-2-infected cells. The EMBO Journal 39, e106267 (2020)https://doi.org/10.15252/embj.2020106267). 
33. T. C. Dakal, SARS-CoV-2 attachment to host cells is possibly mediated via RGD-integrin interaction in a calcium-dependent manner and suggests pulmonary EDTA chelation therapy as a novel treatment for COVID 19. Immunobiology 226, 152021 (2021); published online Epub2021/01/01/ (https://doi.org/10.1016/j.imbio.2020.152021).

34. B. P. Jena, Role of SNAREs in membrane fusion. Cell Fusion in Health and Disease, 13-32 (2011).

35. I. G. Mills, S. Urbé, M. J. Clague, Relationships between EEA1 binding partners and their role in endosome fusion. Journal of Cell Science 114, 1959-1965 (2001).

36. M. Zhang, C. Abrams, L. Wang, A. Gizzi, L. He, R. Lin, Y. Chen, P. J. Loll, J. M. Pascal, J.-f. Zhang, Structural basis for calmodulin as a dynamic calcium sensor. Structure 20, 911-923 (2012).

37. S. Mac Neil, S. W. Walker, J. Seid, S. Tomlinson, Calmodulin in human serum and the specific release of calmodulin from calmodulin-rich platelets. Bioscience Reports 4, 643-650 (1984); published online Epub1984/08/01 (10.1007/BF01121017).

38. S. W. WALKER, S. M. NEIL, J. SEID, S. TOMLINSON, Calmodulin release from human platelets in response to thrombin: presence of calmodulin in serum. Biochemical Society Transactions 13, 234-235 (1985)10.1042/bst0130234).

39. M. I. Colombo, W. Beron, P. D. Stahl, Calmodulin regulates endosome fusion. Journal of Biological Chemistry 272, 7707-7712 (1997).

40. L. S. Maier, D. M. Bers, Role of $\mathrm{Ca} 2+/$ calmodulin-dependent protein kinase $(\mathrm{CaMK})$ in excitation-contraction coupling in the heart. Cardiovascular research 73, 631-640 (2007).

41. J. J. Saucerman, D. M. Bers, Calmodulin mediates differential sensitivity of CaMKII and calcineurin to local Ca2+ in cardiac myocytes. Biophysical journal 95, 4597-4612 (2008).

42. D. Cashman, Dominance of SARS-CoV-2 D614G variant explained by the requirement of COVID-19 for calcium; proximate therapeutic implication (s) for COVID-19. J Clin Immunol Immunother 6, 2 (2020).

43. Y. Huang, C. Yang, X.-f. Xu, W. Xu, S.-w. Liu, Structural and functional properties of SARS-CoV-2 spike protein: potential antivirus drug development for COVID-19. Acta Pharmacologica Sinica 41, 1141-1149 (2020); published online Epub2020/09/01 (10.1038/s41401-020-0485-4).

44. C. Yang, X. Pan, Y. Huang, C. Cheng, X. Xu, Y. Wu, Y. Xu, W. Shang, X. Niu, Y. Wan, Z. Li, R. Zhang, S. Liu, G. Xiao, W. Xu, Drug Repurposing of Itraconazole and Estradiol Benzoate against COVID-19 by Blocking SARS-CoV-2 Spike Protein-Mediated Membrane Fusion. Advanced Therapeutics n/a, 2000224 https://doi.org/10.1002/adtp.202000224).

45. A. L. Lai, J. H. Freed, SARS-CoV-2 Fusion Peptide has a Greater Membrane Perturbating Effect than SARS-CoV with Highly Specific Dependence on $\mathrm{Ca}<\sup >2+</$ sup $>$. bioRxiv, 2021.2001.2004.425297 (2021)10.1101/2021.01.04.425297).

46. G. Khelashvili, A. Plante, M. Doktorova, H. Weinstein, Ca2+-dependent mechanism of membrane insertion and destabilization by the SARS-CoV-2 fusion peptide. Biophysical Journal 120, 1105-1119 (2021); published online Epub2021/03/16/ (https://doi.org/10.1016/j.bpj.2021.02.023).

47. A. D. Davidson, M. K. Williamson, S. Lewis, D. Shoemark, M. W. Carroll, K. J. Heesom, M. Zambon, J. Ellis, P. A. Lewis, J. A. Hiscox, Characterisation of the transcriptome and proteome of SARS-CoV-2 reveals a cell passage induced in-frame deletion of the furin-like cleavage site from the spike glycoprotein. Genome medicine 12, 1-15 (2020). 
48. G. S. Gunaratne, E. Brailoiu, S. He, E. M. Unterwald, S. Patel, J. T. Slama, T. F. Walseth, J. S. Marchant, Essential requirement for JPT2 in NAADP-evoked $\mathrm{Ca}<\sup >2+</$ sup $>$ signaling. Science Signaling 14, eabd5605 (2021)10.1126/scisignal.abd5605).

49. O. H. Petersen, O. V. Gerasimenko, J. V. Gerasimenko, Endocytic uptake of SARS-CoV-2: the critical roles of pH, Ca2+, and NAADP. Function 1, zqaa003 (2020).

50. R. Hu, C. Han, S. Pei, M. Yin, X. Chen, Procalcitonin levels in COVID-19 patients. International journal of antimicrobial agents 56, 106051 (2020).

51. L. A. Austin, H. Heath III, Calcitonin: physiology and pathophysiology. New England Journal of Medicine 304, 269-278 (1981).

52. G. V. Foster, Calcitonin (thyrocalcitonin). New England Journal of Medicine 279, 349-360 (1968).

53. F. Cappellini, R. Brivio, M. Casati, A. Cavallero, E. Contro, P. Brambilla, Low levels of total and ionized calcium in blood of COVID-19 patients. Clinical Chemistry and Laboratory Medicine (CCLM) 58, e171-e173 (2020).

54. A. S. Elham, K. Azam, J. Azam, L. Mostafa, B. Nasrin, N. Marzieh, Serum vitamin D, calcium, and zinc levels in patients with COVID-19. Clinical Nutrition ESPEN, (2021); published online Epub2021/04/18/ (https://doi.org/10.1016/j.clnesp.2021.03.040).

55. W. Osman, F. Al Fahdi, F. Khamis, I. A. Salmi, H. A. Khalili, A. Gokhale, Serum Calcium and Vitamin D levels correlation with severity of COVID-19 in hospitalized patients in Royal Hospital, Oman. International Journal of Infectious Diseases, (2021); published online Epub2021/04/20/ (https://doi.org/10.1016/j.ijid.2021.04.050).

56. N. J. Gittoes, S. Criseno, N. M. Appelman-Dijkstra, J. Bollerslev, E. Canalis, L. Rejnmark, Z. Hassan-Smith, ENDOCRINOLOGY IN THE TIME OF COVID-19: management of calcium metabolic disorders and osteoporosis. European journal of endocrinology 183, G57-G65 (2020).

57. Y. Zhou, T. K. Frey, J. J. Yang, Viral calciomics: interplays between $\mathrm{Ca} 2+$ and virus. Cell calcium 46, 1-17 (2009).

58. F. J. van Kuppeveld, A. S. de Jong, W. J. Melchers, P. H. Willems, Enterovirus protein 2B po (u) res out the calcium: a viral strategy to survive? Trends in microbiology 13, 41-44 (2005).

59. M. Carretero, M. Gómez-Gonzalo, E. Lara-Pezzi, I. Benedicto, J. Aramburu, S. Martinez-Martínez, J. M. Redondo, M. López-Cabrera, The hepatitis B virus X protein binds to and activates the NH2-terminal trans-activation domain of nuclear factor of activated T cells- 1 . Virology 299, 288-300 (2002).

60. I. I. Kruman, A. Nath, M. P. Mattson, HIV-1 protein Tat induces apoptosis of hippocampal neurons by a mechanism involving caspase activation, calcium overload, and oxidative stress. Experimental neurology 154, 276-288 (1998).

61. L. H. Chao, M. M. Stratton, I.-H. Lee, O. S. Rosenberg, J. Levitz, D. J. Mandell, T. Kortemme, J. T. Groves, H. Schulman, J. Kuriyan, A mechanism for tunable autoinhibition in the structure of a human Ca2+/calmodulin-dependent kinase II holoenzyme. Cell 146, 732-745 (2011).

62. A. Hudmon, H. Schulman, Structure-function of the multifunctional Ca2+/calmodulin-dependent protein kinase II. Biochemical Journal 364, 593-611 (2002).

63. P. De Koninck, H. Schulman, Sensitivity of CaM kinase II to the frequency of $\mathrm{Ca} 2+$ oscillations. Science 279, 227-230 (1998).

64. O. S. Rosenberg, S. Deindl, R.-J. Sung, A. C. Nairn, J. Kuriyan, Structure of the autoinhibited kinase domain of CaMKII and SAXS analysis of the holoenzyme. Cell 123, 849-860 (2005). 
65. S. G. Miller, B. L. Patton, M. B. Kennedy, Sequences of autophosphorylation sites in neuronal type II CaM kinase that control Ca2+-independent activity. Neuron 1, 593-604 (1988).

66. T. Meyer, P. I. Hanson, L. Stryer, H. Schulman, Calmodulin trapping by calcium-calmodulin-dependent protein kinase. Science 256, 1199-1202 (1992).

67. G. Lu, Q. Wang, G. F. Gao, Bat-to-human: spike features determining 'host jump'of coronaviruses SARS-CoV, MERS-CoV, and beyond. Trends in microbiology 23, 468-478 (2015).

68. A. C. Walls, Y.-J. Park, M. A. Tortorici, A. Wall, A. T. McGuire, D. Veesler, Structure, function, and antigenicity of the SARS-CoV-2 spike glycoprotein. Cell 181, 281-292. e286 (2020).

69. M. Hussain, N. Jabeen, A. Amanullah, A. A. Baig, B. Aziz, S. Shabbir, F. Raza, N. Uddin, Molecular docking between human TMPRSS2 and SARS-CoV-2 spike protein: conformation and intermolecular interactions. AIMS Microbiol 6, 350-360 (2020)10.3934/microbiol.2020021).

70. T. Tang, M. Bidon, J. A. Jaimes, G. R. Whittaker, S. Daniel, Coronavirus membrane fusion mechanism offers a potential target for antiviral development. Antiviral research 178, 104792 (2020).

71. B. J. Bosch, R. Van der Zee, C. A. De Haan, P. J. Rottier, The coronavirus spike protein is a class I virus fusion protein: structural and functional characterization of the fusion core complex. Journal of virology 77, 8801-8811 (2003).

72. U. Kalathiya, M. Padariya, M. Mayordomo, M. Lisowska, J. Nicholson, A. Singh, M. Baginski, R. Fahraeus, N. Carragher, K. Ball, Highly conserved homotrimer cavity formed by the SARS-CoV-2 spike glycoprotein: a novel binding site. Journal of clinical medicine 9, 1473 (2020).

73. S. Liu, G. Xiao, Y. Chen, Y. He, J. Niu, C. R. Escalante, H. Xiong, J. Farmar, A. K. Debnath, P. Tien, Interaction between heptad repeat 1 and 2 regions in spike protein of SARS-associated coronavirus: implications for virus fusogenic mechanism and identification of fusion inhibitors. The Lancet 363, 938-947 (2004).

74. X. Fan, D. Cao, L. Kong, X. Zhang, Cryo-EM analysis of the post-fusion structure of the SARS-CoV spike glycoprotein. Nature Communications 11, 3618 (2020); published online Epub2020/07/17 (10.1038/s41467-020-17371-6).

75. L. Zhang, L. Du, B. Poovaiah, Calcium signaling and biotic defense responses in plants. Plant signaling \& behavior 9, e973818 (2014).

76. S. Xia, Y. Zhu, M. Liu, Q. Lan, W. Xu, Y. Wu, T. Ying, Fusion mechanism of 2019-nCoV and fusion inhibitors targeting HR1 domain in spike protein. 17, 765-767 (2020); published online EpubJul (10.1038/s41423-020-0374-2).

77. C. Liu, Y. Feng, F. Gao, Q. Zhang, M. Wang, Characterization of HCoV-229E fusion core: implications for structure basis of coronavirus membrane fusion. Biochemical and biophysical research communications 345, 1108-1115 (2006).

78. S. Buonvino, S. Melino, New Consensus pattern in Spike CoV-2: potential implications in coagulation process and cell-cell fusion. Cell Death Discovery 6, 134 (2020); published online Epub2020/11/27 (10.1038/s41420-020-00372-1).

79. K. W. Chang, Y. Sheng, J. L. Gombold, Coronavirus-induced membrane fusion requires the cysteine-rich domain in the spike protein. Virology 269, 212-224 (2000).

80. E. C. Bos, L. Heijnen, W. Luytjes, W. J. Spaan, Mutational analysis of the murine coronavirus spike protein: effect on cell-to-cell fusion. Virology 214, 453-463 (1995). 
81. A. Shulla, T. Gallagher, Role of spike protein endodomains in regulating coronavirus entry. Journal of Biological Chemistry 284, 32725-32734 (2009).

82. A. Singh, P. Kanwar, A. K. Yadav, M. Mishra, S. K. Jha, V. Baranwal, A. Pandey, S. Kapoor, A. K. Tyagi, G. K. Pandey, Genome-wide expressional and functional analysis of calcium transport elements during abiotic stress and development in rice. The FEBS journal 281, 894-915 (2014).

83. M. Geisler, K. B. Axelsen, J. F. Harper, M. G. Palmgren, Molecular aspects of higher plant P-type Ca2+-ATPases. Biochimica et Biophysica Acta (BBA)-Biomembranes 1465, 52-78 (2000).

84. K. M. K. Huda, S. Yadav, M. S. A. Banu, D. K. Trivedi, N. Tuteja, Genome-wide analysis of plant-type II Ca2+ ATPases gene family from rice and Arabidopsis: potential role in abiotic stresses. Plant Physiology and Biochemistry 65, 32-47 (2013).

85. J. F. Harper, B. Hong, I. Hwang, H. Q. Guo, R. Stoddard, J. F. Huang, M. G. Palmgren, H. Sze, A novel calmodulin-regulated Ca2+-ATPase (ACA2) from Arabidopsis with an N-terminal autoinhibitory domain. The Journal of biological chemistry 273, 1099-1106 (1998); published online Epub ( ).

86. A. Sultana, P. Kallio, A. Jansson, J.-S. Wang, J. Niemi, P. Mäntsälä, G. Schneider, Structure of the polyketide cyclase SnoaL reveals a novel mechanism for enzymatic aldol condensation. The EMBO journal 23, 1911-1921 (2004); published online Epub2004/05// (

87. J. L. Gifford, M. P. Walsh, H. J. Vogel, Structures and metal-ion-binding properties of the Ca2+-binding helix-loop-helix EF-hand motifs. The Biochemical journal 405, 199-221 (2007); published online EpubJul 15 (10.1042/bj20070255).

88. B. Schwaller, Cytosolic Ca2+ buffers. Cold Spring Harbor perspectives in biology 2, a004051 (2010); published online EpubNov (10.1101/cshperspect.a004051).

89. B. E. Finn, S. Forsén, The evolving model of calmodulin structure, function and activation. Structure 3, 7-11 (1995); published online EpubJan 15 (10.1016/s0969-2126(01)00130-7).

90. C. Ban, B. Ramakrishnan, K. Y. Ling, C. Kung, M. Sundaralingam, Structure of the recombinant Paramecium tetraurelia calmodulin at 1.68 A resolution. Acta crystallographica. Section D, Biological crystallography 50, 50-63 (1994); published online EpubJan 1 (10.1107/s0907444993007991).

91. N. K. Vyas, M. N. Vyas, F. A. Quiocho, A novel calcium binding site in the galactose-binding protein of bacterial transport and chemotaxis. Nature 327, 635-638 (1987).

92. T. L. Wingo, V. N. Shah, M. E. Anderson, T. P. Lybrand, W. J. Chazin, J. R. Balser, An EF-hand in the sodium channel couples intracellular calcium to cardiac excitability. Nature structural \& molecular biology 11, 219-225 (2004).

93. S. K. Hanks, A. M. Quinn, T. Hunter, The protein kinase family: conserved features and deduced phylogeny of the catalytic domains. Science 241, 42-52 (1988); published online EpubJul 1 (10.1126/science.3291115).

94. G. Manning, G. D. Plowman, T. Hunter, S. Sudarsanam, Evolution of protein kinase signaling from yeast to man. Trends in biochemical sciences 27, 514-520 (2002); published online EpubOct (10.1016/s0968-0004(02)02179-5).

95. X. Robert, P. Gouet, Deciphering key features in protein structures with the new ENDscript server. Nucleic Acids Research 42, W320-W324 (2014)10.1093/nar/gku316).

96. L. De Haro, S. Quetglas, C. Iborra, C. Lévêque, M. Seagar, Calmodulin-dependent regulation of a lipid binding domain in the v-SNARE synaptobrevin and its role in vesicular fusion. Biology of the Cell 95, 459-464 (2003). 
97. M. Leabu, Membrane fusion in cells: molecular machinery and mechanisms. Journal of Cellular and Molecular Medicine 10, 423-427 (2006)https://doi.org/10.1111/j.1582-4934.2006.tb00409.x).

98. B. P. Jena, Membrane fusion: role of SNAREs and calcium. Protein and peptide letters 16, 712-717 (2009).

99. C.-Y. Tai, Q. R. Smith, S. I. Rapoport, Calcium influxes into brain and cerebrospinal fluid are linearly related to plasma ionized calcium concentration. Brain research 385, 227-236 (1986).

100. J. Kozik-Jaromin, V. Nier, U. Heemann, B. Kreymann, J. Böhler, Citrate pharmacokinetics and calcium levels during high-flux dialysis with regional citrate anticoagulation. Nephrology Dialysis Transplantation 24, 2244-2251 (2009).

101. B. W. Zweifach, in Cold Spring Harbor Symposia on Quantitative Biology. (Cold Spring Harbor Laboratory Press, 1940), vol. 8, pp. 216-223.

102. A. R. Means, in Principles of molecular regulation. (Springer, 2000), pp. 187-204.

103. T. M. Clausen, D. R. Sandoval, C. B. Spliid, J. Pihl, H. R. Perrett, C. D. Painter, A. Narayanan, S. A. Majowicz, E. M. Kwong, R. N. McVicar, B. E. Thacker, C. A. Glass, Z. Yang, J. L. Torres, G. J. Golden, P. L. Bartels, R. N. Porell, A. F. Garretson, L. Laubach, J. Feldman, X. Yin, Y. Pu, B. M. Hauser, T. M. Caradonna, B. P. Kellman, C. Martino, P. L. S. M. Gordts, S. K. Chanda, A. G. Schmidt, K. Godula, S. L. Leibel, J. Jose, K. D. Corbett, A. B. Ward, A. F. Carlin, J. D. Esko, SARS-CoV-2 Infection Depends on Cellular Heparan Sulfate and ACE2. Cell 183, 1043-1057.e1015 (2020).

104. A. N. Teien, U. Abildgaard, M. Höök, The anticoagulant effect of heparan sulfate and dermatan sulfate. Thrombosis Research 8, 859-867 (1976); published online Epub1976/06/01/ (https://doi.org/10.1016/0049-3848(76)90014-1).

105. W. Liu, H. Li, COVID-19: attacks the 1-beta chain of hemoglobin and captures the porphyrin to inhibit human heme metabolism. ChemRxiv. Preprint. chemrxiv.11938173.v9, (2020); published online Epub2020.7.13 (

106. M. Madjid, D. Vela, H. Khalili-Tabrizi, S. W. Casscells, S. Litovsky, Systemic infections cause exaggerated local inflammation in atherosclerotic coronary arteries: clues to the triggering effect of acute infections on acute coronary syndromes. Texas heart institute journal 34, 11 (2007). 